\title{
Protective effects of genistein and melatonin on mouse liver injury induced by whole-body ionising radiation
}

\author{
GONCA HANEDAN USLU ${ }^{1}$, EMINE CANYILMAZ ${ }^{2}$, LASIF SERDAR $^{1}$ and ŞAFAK ERSÖZ ${ }^{3}$ \\ ${ }^{1}$ Department of Radiation Oncology, Health Sciences University Kanuni Training and Research Hospital, \\ Trabzon 61250; Departments of ${ }^{2}$ Radiation Oncology and ${ }^{3}$ Medical Pathology, \\ Faculty of Medicine, Karadeniz Technical University, Trabzon 61080, Turkey
}

Received May 4, 2018; Accepted December 4, 2018

DOI: $10.3892 / \mathrm{mco} .2018 .1790$

\begin{abstract}
The aim of the present study was to investigate the effectiveness of melatonin and genistein in preventing radiation therapy (RT)-induced liver injury in mice. A total of 70 Swiss Albino male mice were divided into 7 equal groups ( $n=10 /$ group) as follows: Melatonin (M group, G3), genistein (G group, G4), polyethylene glycol-400 (P group, G5), RT only (RT group, G2) and sham irradiation (C group, G1). RT plus genistein (RT+G group, G7) and RT plus melatonin (RT+M group, G6) were the co-treatment groups. Firstly, hepatic tissue damage was induced in mice via exposure to a single dose of 6-Gy irradiation. RT was performed with a cobalt-60 teletherapy machine $(80 \mathrm{~cm}$ fixed source-to-surface distance, 2.5-cm depth). Melatonin was processed $(100 \mathrm{mg} / \mathrm{kg}$, intraperitoneal) $30 \mathrm{~min}$ before and genistein was administered (200 mg/kg, SC) one day prior to the single dose of irradiation. Six months following irradiation, all mice were sacrificed. The degree of liver injury was measured using histological liver sections. Liver injury was significantly worse in the RT group than in the control group $(\mathrm{C}$; RT vs. $\mathrm{C}$; $\mathrm{P}<0.05)$; however, liver injury decreased following co-treatment with melatonin or genistein vs. RT alone $(\mathrm{RT}+\mathrm{M}$ and $\mathrm{RT}+\mathrm{G}$ vs. RT; $\mathrm{P}<0.05)$. No difference was observed between the RT+M and $\mathrm{RT}+\mathrm{G}$ groups $(\mathrm{P}>0.05)$. The present study revealed that melatonin and genistein administration prior to irradiation protects mice against liver injury, which may have therapeutic implications for RT-induced injuries.
\end{abstract}

Correspondence to: Dr Gonca Hanedan Uslu, Department of Radiation Oncology, Health Sciences University Kanuni Training and Research Hospital, Numune Campus, İnönü Street, Ortahisar, Trabzon 61250, Turkey

E-mail: drgoncahanedanuslu@hotmail.com

Key words: genistein, hepatotoxicity, melatonin, mice, radiation, radioprotection

\section{Introduction}

Radiation therapy (RT) is one of the most crucial treatments in cancer therapy and the mainstay treatment in patients with abdominopelvic cancer $(1,2)$. Modern RT techniques have increased cure and locoregional control rates in the treatment of cancer. However, these techniques have not ensured the complete removal of the toxic effects of RT on normal tissues. Because it is not always possible to treat RT-related short- and long-term adverse effects, agents that are able to prevent the development of adverse effects have gained importance. It is possible to prevent RT-induced destructive changes in normal cells caused by free radicals (FRs) using FR scavenger agents.

Clinical and experimental histopathological studies have demonstrated that abdominal RT may lead to hepatic lesions. In particular, in the RT of gastric and pancreatic cancers, lymphomas as well as cancers of the abdominal region and right lung, liver tissue is inevitably and adversely affected by radiation exposure. Radiation hepatitis which may develop can clinically lead to suprahepatic vein obstruction or Budd-Chiari syndrome with ensuing mortal outcomes. RT-induced hepatitis is not characterised by neutrophilic infiltration as hepatitis related to other causes, but a veno-occlusive condition is observed. The first observed morphological change in veno-occlusive disease which may originate from the liver as a whole, a lobe or a lobule is generally congestion starting from the central region of the lobule and associated with yellow necrotic focus $(3,4)$.

Ionising radiation induces an increase in oxygen FRs in the biological system. These radicals affect DNA, proteins and membrane lipids of cells and lead to cell damage. Oxygen FRs occurring as a result of radiation exposure affect antioxidant defence mechanisms adversely. This negative effect decreases the intracellular glutathione (GSH) level; it also decreases the activities of intracellular antioxidant enzymes, particularly superoxide dismutase (SOD), catalase and GSH peroxidase (GSH-Px) (5). The oxidative translation of unsaturated fatty acids to metabolic products, including malondialdehyde (MDA) and lipid peroxides, is an FR process called RT-induced lipid peroxidation (6).

One method which may be used to decrease the damage induced by RT via these mechanisms is a restriction of the dose used. However, this method is not applicable because it may 
adversely affect tumoral response. Another most frequently emphasised method is the use of radioprotective agents. There are many radioprotective agents, among which the most commonly used agent for head and neck cancers today is amifostine. In addition, there are several radioprotective agents, such as melatonin, which is natural, and thiol compounds, other compounds containing -SH radical, pharmacological agents and other radioprotective agents (7). Melatonin is the best known among natural radioprotective agents. Melatonin was found to be a potent FR scavenger in 1993. Since then, over 800 studies have directly or indirectly confirmed this observation. Based on the analyses of structure-activity relationships, the indole moiety of the melatonin molecule is the reactive centre of interaction with oxidants due to its high resonance stability and very low activation energy barrier towards FR reactions (8). Hence, melatonin is of high interest. Another radioprotective agent that has become popular in recent years is genistein. Genistein is an isoflavone found in soybean. It has known radioprotective effects in mice (9). For these reasons, this study's aim was to investigate the effectiveness of radioprotective agents melatonin and genistein in preventing RT-induced liver injury in mice.

\section{Materials and methods}

Study design. In this experimental study, It was designed with a total of 70 mice. Swiss Albino male mice (10-12-week-old, weighing $25 \pm 2 \mathrm{~g}$ ) were purchased from the Center for Laboratory Animals at the Karadeniz Technical University (Trabzon, Turkey) (2013/28). All the mice were acclimatised upon arrival, and representative animals were screened for evidence of disease. The Institutional Animal Care and Use Committee at Karadeniz Technical University (Trabzon, Turkey) approved the protocol used in the present study. Animals were housed four per cage in a controlled animal holding room with a 12/12-h light/dark cycle; temperature $\left(22^{\circ} \mathrm{C} \pm 1^{\circ} \mathrm{C}\right)$ and relative humidity were continually monitored to provide standard laboratory conditions. Food and water were provided ad libitum.

Mice were divided into seven groups comprising ten animals each. Group I (C): Control was defined as the control group, and mice in this group were sham-irradiated; Group II (RT): Whole-body RT was administered; Group III (M): Melatonin was administered; Group IV (G): Genistein was administered; Group V (P): Polyethylene glycol (PEG)-400 was administered; Group VI (RT+M): Melatonin was administered 30 min before RT; and Group VII (RT+G): Genistein was administered $24 \mathrm{~h}$ before RT.

The animals were sacrificed 24 weeks later. As an end point, the extent of spot necrosis for each mouse was quantified with image analysis of histological sections of the liver (10).

Irradiation protocol. Before whole-body irradiation, the animals were anaesthetised with intraperitoneal (i.p.) injections of $90 \mathrm{mg} / \mathrm{kg}$ of ketamine and $10 \mathrm{mg} / \mathrm{kg}$ of xylazine. Subsequently, the animals were placed on a straphore in the prone position by taping their extremities. Correct positioning of the fields was controlled for each mouse via a therapy simulator. A single dose of 6-Gy $\gamma$-radiation was selected according to previous studies $(11,12)$. Mice in the RT, $\mathrm{RT}+\mathrm{M}$ and $\mathrm{RT}+\mathrm{G}$ groups were irradiated with a cobalt-60 teletherapy machine from a source-to-surface distance of $80 \mathrm{~cm}$. A single dose of 6-Gy $\gamma$-radiation was delivered to the whole body at a dose rate of $47.50 \mathrm{~Gy} / \mathrm{min}$. The dose was calculated for the central axis at a depth of $2.5 \mathrm{~cm}$.

Melatonin and genistein protocols. For the mice in the $\mathrm{M}$ and $\mathrm{RT}+\mathrm{M}$ groups, melatonin(Melatonin Crystalline; Sigma-Aldrich, St. Louis, MO, USA) was prepared at a $1 \%$ concentration by dissolving in ethanol and diluting in $0.9 \%$ sodium chloride; this was administered at a dose of $100 \mathrm{mg} / \mathrm{kg}$ i.p. $30 \mathrm{~min}$ prior to exposure to radiation. The selection of a 30-min interval between the melatonin administration and exposure to radiation was based on two previous studies in animals $(13,14)$ and human volunteers $(15,10)$. Genistein and PEG, of molecular weight 400, were obtained from Sigma-Aldrich. Genistein was solubilised in PEG-400 on the day of the experiment using $20 \mathrm{sec}$ of sonication (Heat Systems-Ultrasonics Inc., Plainview, NY, USA). Genistein was administered at a dose of $100 \mathrm{mg} / \mathrm{kg}$ subcutaneously (s.c.) $24 \mathrm{~h}$ before being exposed to radiation. Of note, $0.9 \%$ sodium chloride was prepared at an equal volume with melatonin, and the remaining procedure was applied identically for mice in the $\mathrm{G}$ and $\mathrm{RT}+\mathrm{G}$ groups. PEG-400 was prepared at an equal volume with genistein, and the rest of the procedure was applied identically to group $\mathrm{P}$ mice. The selection of a 24-h interval between genistein administration and exposure to radiation was based on one earlier study in animals $(16,17)$.

Morphological study and light microscopy. The animals were anaesthetised and sacrificed by cervical dislocation 24 weeks after the start of irradiation. The liver was excised and fixed in a 10\% formaldehyde solution and embedded in paraffin for light microscopic examination. One transverse section of the liver was taken using vertical sections. The slices obtained were stained with hematoxylin and eosin to evaluate the fibrosis in the liver. The same pathologist histopathologically examined and graded all liver tissues. Grade 0 , absence of tissue damage; Grade 1, the presence of one to four necrotic foci within the field of vision under 10x magnification; Grade 2, more than four foci within the field of vision under 10x magnification (18).

Statistical analysis. Ordinal/nominal data were compared using Chi-square test (and for smaller data, Fisher's exact test). In the evaluation of data, fitness of variables to a normal distribution was examined using visual (histograms and probability graphics) and analytical methods (Kolmogorov-Smirnov tests). Following examination of the distribution of variables, parametric interval data were analysed using the significance of the difference between two means, Student's t-test or one-way analysis of variance with Tukey's post hoc test, whereas non-parametric interval data were examined using Mann-Whitney U test or Kruskal-Wallis test. Levene's test was used to assess the homogeneity of variances. Type 1 errors of $<5 \%$ were accepted as statistically significant. All of the statistical analyses were performed using SPSS version 13 (SPSS, Inc., Chicago, IL, USA).

\section{Results}

In the histopathological examination of mice liver, mice without any liver necrosis were scored as Grade 0 (Fig. 1). 
Grade 2 mice were those with more than four foci of hepatic necrosis under 10x magnification (Fig. 2).

The histopathological analysis revealed that the livers of the mice in the control group were evaluated as Grade $0(n=3$; $30 \%)$, Grade $1(n=5 ; 50 \%)$ and Grade $2(n=2 ; 20 \%)$. In all mice $(100 \%)$ in the RT group, hepatic necrosis was observed. The livers of the mice in the $\mathbf{M}$ group were evaluated as Grade $0(n=2 ; 20 \%)$ and Grade $1(n=8 ; 80 \%)$. In the $\mathrm{G}$ group, the livers of the mice were evaluated as Grade $0(n=1 ; 10 \%)$, Grade $1(n=7 ; 70 \%)$ and Grade $2(n=2 ; 20 \%)$. The livers of the mice in the PEG group were evaluated as Grade $1(\mathrm{n}=8$; $80 \%)$ and Grade $2(n=2 ; 20 \%)$. The livers of the mice in the $\mathrm{RT}+\mathrm{M}$ group were evaluated as Grade $0(\mathrm{n}=4 ; 40 \%)$, Grade 1 $(n=5 ; 50 \%)$ and Grade $2(n=1 ; 10 \%)$. The livers of the mice in the $\mathrm{RT}+\mathrm{G}$ group were evaluated as Grade $1(\mathrm{n}=7 ; 70 \%)$ and Grade $2(n=3 ; 30 \%)$. The histopathological analysis of the liver specimens did not reveal any evidence of Grade 3 hepatic necrosis. Grades of hepatic necrosis in all groups are presented. A statistically significant difference was observed between groups $(\mathrm{P}<0.001)$.

A statistically significant difference was observed between the $\mathrm{C}$ and RT groups regarding grades of hepatic necrosis $(\mathrm{P}=0.001)$. Hepatic necrosis was more severe in the RT group relative to the $\mathrm{C}$ group. Statistically significant differences were observed between the RT group and the $\mathrm{G}(\mathrm{P}<0.001), \mathrm{M}$ $(\mathrm{P}<0.001)$ or $\mathrm{PEG}(\mathrm{P}<0.001)$ groups. In the RT group, hepatic necrosis was more destructive when compared with all other groups (Fig. 3).

No statistically significant difference was observed in histopathological analysis between the $\mathrm{C}$ group and the $\mathrm{G}, \mathrm{M}$, PEG, RT+G and RT+M groups ( $\mathrm{P}>0.05)$.

A statistically significant difference was seen in the grades of hepatic necrosis between the RT and RT+G groups $(\mathrm{P}=0.003)$. When compared with the isolated RT group (Group II), a marked decrease in RT-related liver injury scores was observed in the RT+G group (Fig. 4).

A statistically significant difference was seen between the RT and RT+M groups regarding grades of hepatic injury $(\mathrm{P}<0.001)$. When compared with the isolated RT group, the concomitant administration of melatonin and RT decreased liver injury scores (Fig. 5).

In conclusion, when compared with the isolated use of RT, the concomitant use of genistein or melatonin with RT demonstrated hepatoprotection against harmful effects of RT by decreasing the hepatotoxicity of RT. However, a statistically significant difference was not observed between the $\mathrm{RT}+\mathrm{G}$ and $\mathrm{RT}+\mathrm{M}$ groups $(\mathrm{P}>0.05)$. Thus, genistein has similar hepatoprotective effects to those of melatonin.

\section{Discussion}

In the present study, liver injury in mice induced by RT was determined. As is known, oxidative stress in tissues increases and antioxidant enzyme levels decrease with exposure to ionising radiation. As a result of these mechanisms, organ injuries occur. As a natural phenomenon, many normal tissues within the field of RT are injured. The liver is also one of the most frequently injured organs during RT performed for cancers of the abdominal region. In addition, the common combination of chemotherapeutics and radiation for any

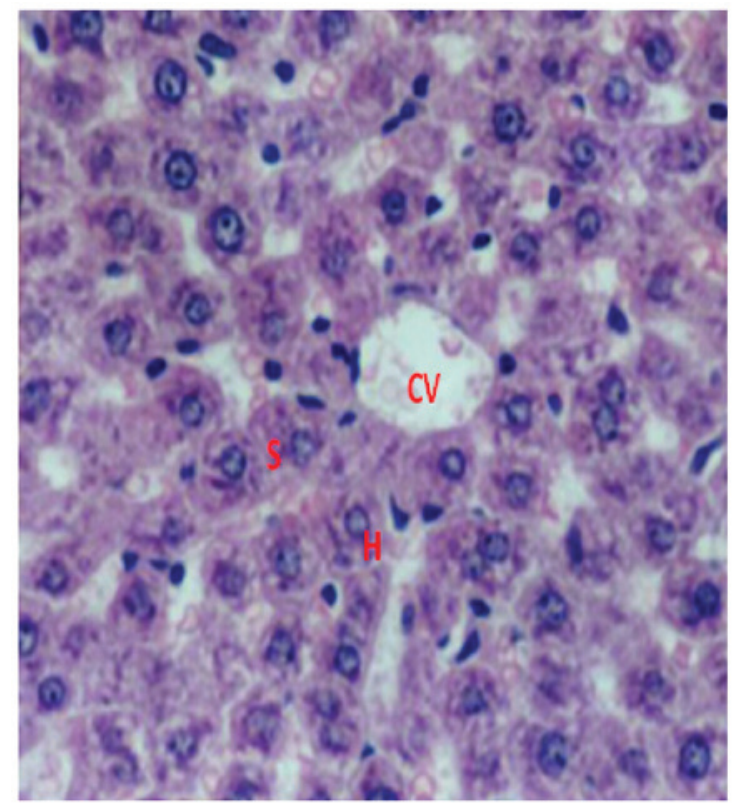

Figure 1. Grade 0 histopathological appearance of hepatic necrosis (hematoxylin and eosin; magnification, x50). $\mathrm{CV}$, central vein; $\mathrm{H}$, hepatocyte; $\mathrm{S}$, sinusoids.

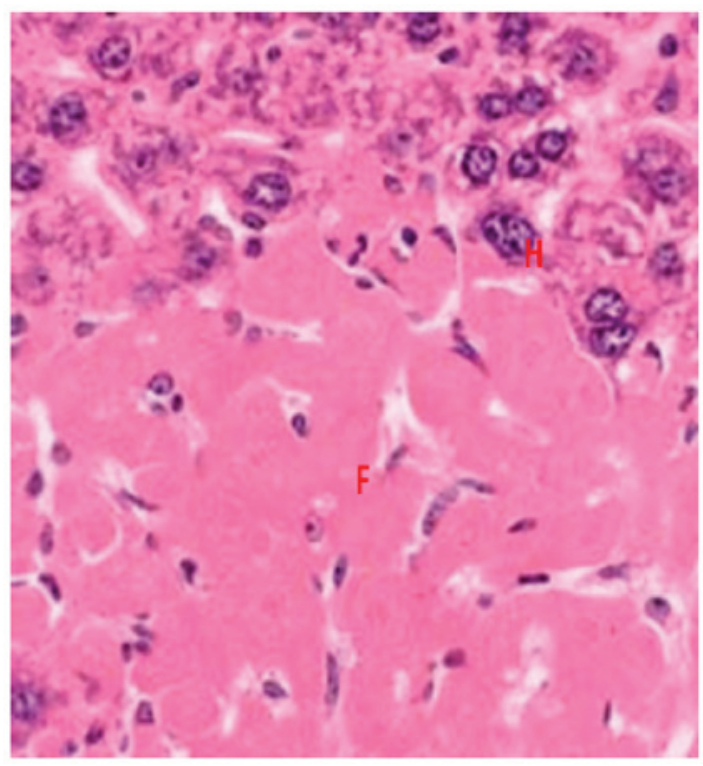

Figure 2. Grade 2 histopathological appearance of hepatic necrosis (hematoxylin and eosin; magnification, $\mathrm{x} 50$ ). F, fibrosis; $\mathrm{H}$, hepatocyte.

number of cancer treatments that involve irradiating the abdomen presents patients with a potential risk of unexpected liver toxicity as a result of $\mathrm{O}_{2}$ generation. When compared with other organs, the liver is a relatively more radiosensitive organ, and measurable changes in metabolic functions as well as in the histopathological structure of this organ are observed following radiation exposure (8). In this study, we were able to apply only one dose of radiotherapy. Because 1 thought clinical conditions were not suitable for fractionating radiotherapy, but that mice could develop pancytopenia and ex as a result.

Many radioprotective agents can be used against this harmful mechanism of RT. Amifostine, melatonin, a, c, e 
12

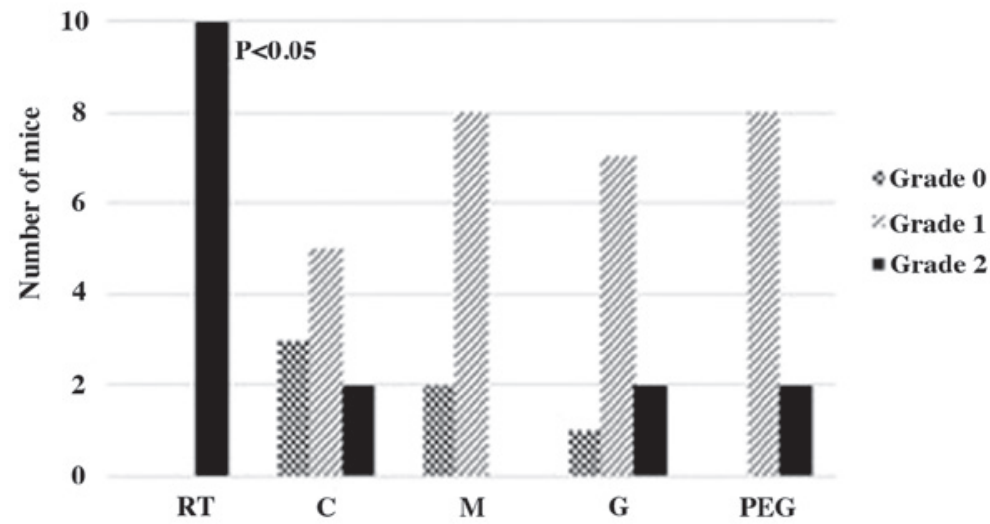

Figure 3. Histopathological grades of hepatic necrosis determined in all groups. RT, radiation therapy; C, sham irradiation (control); M, melatonin; G, genistein; PEG, polyethylene glycol-400.

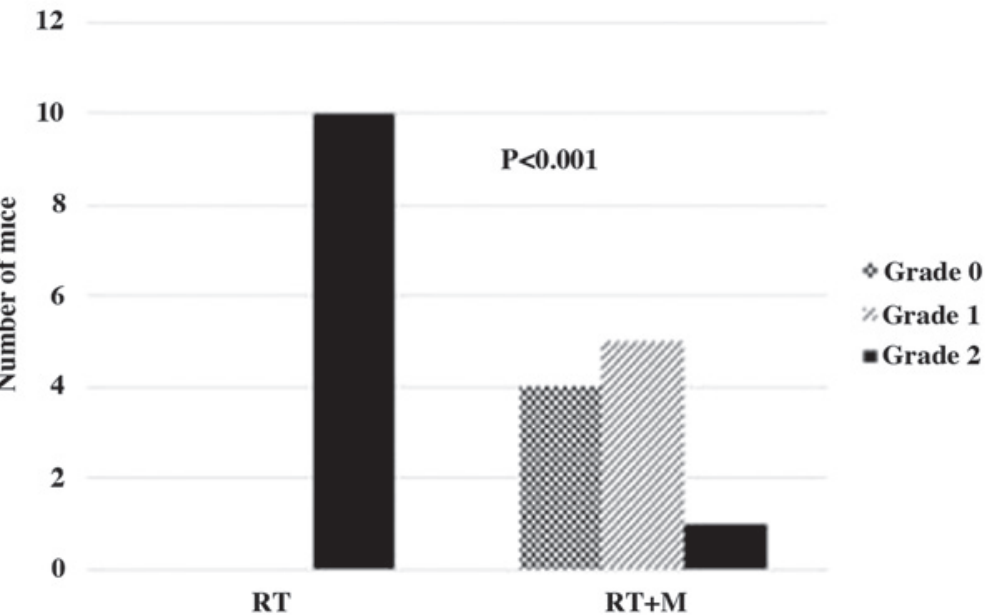

Figure 4. Histopathological grades of hepatic necrosis in the RT and RT+M groups. RT, radiation therapy; M, melatonin.

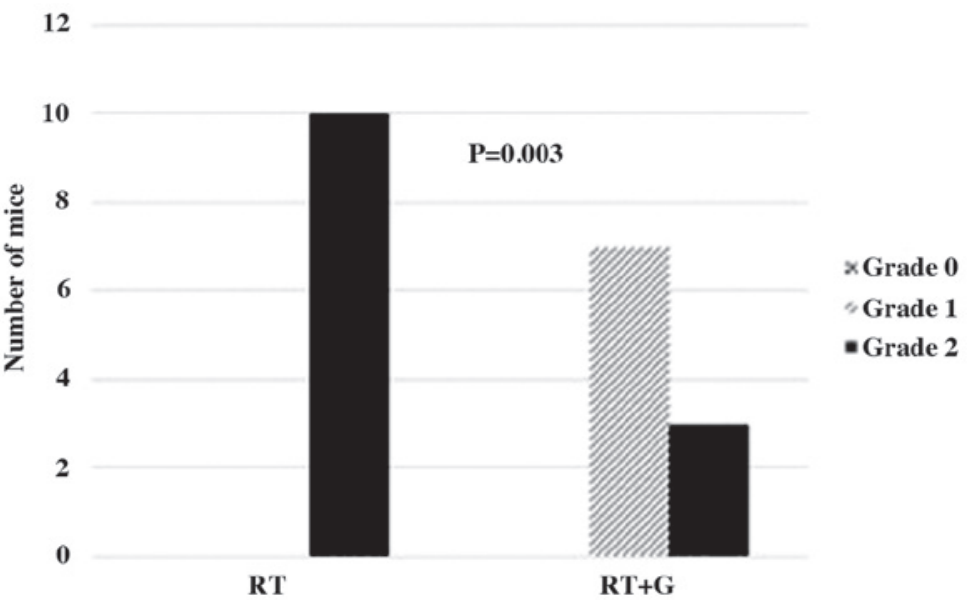

Figure 5. Histopathological grades of hepatic necrosis in the RT and RT+G groups. RT, radiation therapy; G, genistein.

b-carotene vitamins, thiol compounds, other compounds containing-SH radical, pharmacological agents and other radioprotective agents are among these agents (7). Radioprotective agents are compounds administered before exposure to ionising radiation to reduce its damaging effects, including RT-induced lethality (19). The ideal radioprotectant would be non-toxic, would not degrade performance and would be effective after a single administration, particularly 
when entry is required into an area with potential external radiation hazards. Many studies have demonstrated that melatonin, amifostine, $\mathrm{N}$-acetylcysteine and genistein can be used to this end (20-22).

Melatonin (N-acetyl-5-methoxytryptamine) is a hormone secreted by the pineal gland in the dark and plays a role in the regulation of many biological functions, including sleep, reproduction, circadian rhythm and immunity. It has dual effects: i) Direct antioxidative effect: As reported in many studies, melatonin detoxifies FRs that may lead to oxidative stress, including $\mathrm{HO}, \mathrm{H}_{2} \mathrm{O}_{2}, \mathrm{O}_{2}, \mathrm{HOCl}$, $\mathrm{NO}$ and $\mathrm{ONOO}$, thus inhibiting their harmful effects on biomolecules. Comparative studies conducted to determine the potency of various antioxidants have demonstrated that melatonin is one of the most potent antioxidants; and ii) Antioxidant enzyme-mediated effect: It has been reported that pharmacological and probably physiologic levels of melatonin increase gene expressions or activities of some antioxidant enzymes, such as SOD, GSH-Px, GSSG-Rd, glucose-6-phosphate dehydrogenase and g-glutamylcysteine synthetase, and through these pathways, melatonin suppresses oxidative stress (23). The radioprotective effect of melatonin was first reported in vitro by Vijayalaxmi et al (24). Previous studies have revealed that melatonin ameliorates RT-induced injury in the eye lens, spinal cord, brain, liver, spleen, kidney, lung, colon and ileum (25-31). In in vivo studies, doses of $10-250 \mathrm{mg} / \mathrm{kg}$ of melatonin have been tested (32). In the present study, $100 \mathrm{mg} / \mathrm{kg}$ of melatonin was administered i.p., which is consistent with the literature.

Genistein (4',5,7-trihydroxyisoflavone) is an isoflavone found in soybean. Isoflavones may prevent oxidative DNA damage by directly affecting FRs or antioxidant scavenger enzymes. Genistein is known as a compound with the highest antioxidant activity among isoflavones (9). It prevents tumoral growth by inhibiting the activities of DNA topoisomerase II and thyroxine and empowers the immune system. Genistein's beneficial effects on breast cancer, prostate cancer, cardiovascular diseases, hypercholesterolemia and osteoporosis are already known. Its antioxidant properties, suppressing effects on radicals, anti-inflammatory features, antiangiogenetic and antitumoural activities in addition to its antioxidative and radioprotective characteristics in the liver and haematopoietic system have been demonstrated in nimal experiments $(17,33)$. Landauer et al (16) showed that in adult mice, peroral genistein was radioprotective and also decreased the incidence of peripheral micronucleated reticulocytes receiving a sublethal dose of RT.

The present study has some limitations. The addition of pre- and post-treatment liver weight as well as MDA and GSH levels in the study would be more useful for readers to compare liver function between treatments and to understand the potent protective effect of these two compounds. If we could apply more than one dose instead of a single dose, it would increase the strength of the study. Biochemical parameters showing liver damage are investigated and another dose may be used in which repeated doses are used. In another study, the dose effect can be investigated by giving $250 \mathrm{mg} / \mathrm{kg}$ instead of $100 \mathrm{mg} / \mathrm{kg}$. Besides, the results were evaluated by a single histopathologist, which is another limitation of this study.

There was no significant difference between 10 and $250 \mathrm{mg} / \mathrm{kg}$ results. Since there was no financial support for the study, he had to consider the cost. Based on the knowledge that the results were not so different, we used $100 \mathrm{mg} / \mathrm{kg}$ dose to be more economical.

At the end of the six-month follow-up, light microscopic studies showed that in the RT+M and RT+G groups, mice liver was healthier than that in the RT only group. Genistein and melatonin may be used with equal effectiveness to relieve hepatic tissue damage induced by ionising radiation. This information may provide an insight for studies to be performed on the protection against liver injury. The present study can be repeated by including a greater number of samples and measuring biochemical parameters indicating liver toxicity. Further experimental and clinical studies wherein biochemical parameters indicating liver toxicity are measured, RT+PEG is added and a greater number of samples is included should be performed. This study is the first study to compare two items for liver protection in the literature.

\section{Acknowledgements}

Not applicable.

Funding

No funding was received.

\section{Availability of data and materials}

All data generated or analyzed during this study are included in this published article.

\section{Authors' contributions}

GHU conceived and designed the study, performed the experiments, and assessed and interpreted the data. EC performed the experiments and interpreted the data. LS performed the experiments and statistical analysis. ŞE collected and analyzed the pathological data.

\section{Ethics approval and consent to participate}

The Institutional Animal Care and Use Committee at Karadeniz Technical University (Trabzon, Turkey) approved the protocol used in the present study.

\section{Patient consent for publication}

Not applicable.

\section{Competing interests}

The authors declare that they have no competing interests.

\section{References}

1. Rubin P, Johnston CJ, Williams JP, McDonald S and Finkelstein JN: A perpetual cascade of cytokines postirradiation leads to pulmonary fibrosis. Int J Radiat Oncol Biol Phys 33: 99-109, 1995.

2. Zheng H, Wang J, Koteliansky VE, Gotwals PJ and Hauer-Jensen M: Recombinant soluble transforming growth factor beta type II receptor ameliorates radiation enteropathy in mice. Gastroenterology 119: 1286-1296, 2000.

3. Fajardo LF: Pathology of radiation injury. New York: Masson Publishers, pp27-30, 1982. 
4. Reed GB Jr and CoxA J Jr: The human liver after radiation injury. A form of veno-occlusive disease. Am J Pathol 48: 597-611, 1966.

5. Karbownik M and Reiter RJ: Antioxidative effects of melatonin in protection against cellular damage caused by ionizing radiation. Proc Soc Exp Biol Med 225: 9-22, 2000.

6. Sies H: Oxidative stress: A concept in redox biology and medicine. Redox Biol 4: 180-183, 2015.

7. Varanda EA and Tavares DC: Radioprotection: Mechanisms and radioprotective agents including honeybee venom. J Venom Anim Toxins 4: 5-21, 1998

8. Berbee M, Fu Q, Boerma M, Pathak R, Zhou D, Kumar KS and Hauer-Jensen M: Reduction of radiation-induced vascular nitrosative stress by the vitamin E analog gammatocotrienol: Evidence of a role for tetrahydrobiopterin. Int J Radiat Oncol Biol Phys 79: 884-891, 2011.

9. Knight DC and Eden JA: A review of the clinical effects of phytoestrogens. Obstet Gynecol 87: 897-904, 1996.

10. Khan S, Adhikari JS, Rizvi MA and Chaudhury NK Radioprotective potential of melatonin against ${ }^{60} \mathrm{Co} \gamma$-ray-induced testicular injury in male C57BL/6 mice. J Biomed Sci 22: 61, 2015.

11. Kaldir M, Cosar-Alas R, Cermik TF, Yurut-Caloglu V, Saynak M, Altaner S, Caloglu M, Kocak Z, Tokatli F, Türe M, et al: Amifostine use in radiation-induced kidney damage. Preclinical evaluation with scintigraphic and histopathologic parameters. Strahlenther Onkol 184: 370-375, 2008.

12. Cosar R, Yurut-Caloglu V, Eskiocak S, Ozen A, Altaner S, Ibis K, Turan N, Denizli B, Uzal C, Saynak M, et al: Radiation-induced chronic oxidative renal damage can be reduced by amifostine. Med Oncol 29: 768-775, 2012.

13. Anwar MM and Moustafa MA: The effect of melatonin on eye lens of mice exposed to ultraviolet radiation. Comp Biochem Physiol C Toxicol Pharmacol 129: 57-63, 2001.

14. Gibbs FP and Vriend J: The half-life of melatonin elimination from rat plasma. Endocrinology 109: 1796-1798, 1981.

15. Wetterberg L, Eriksson O, Friberg Y and Vangbo B: A simplified radioimmunoassay for melatonin and its application to biological fluids. Preliminary observations on the half-life of plasma melatonin in man. Clin Chim Acta 86: 169-177, 1978.

16. Landauer MR, Srinivasan V and Seed TM: Genistein treatment protects mice from ionizing radiation injury. J Appl Toxicol 23: 379-385, 2003

17. Singh VK, Grace MB, Parekh VI, Whitnall MHand Landauer MR: Effects of genistein administration on cytokine induction in whole-body gamma irradiated mice. Int Immunopharmacol 9: 1401-1410, 2009

18. Goodman ZD: Grading and staging systems for inflammation and fibrosis in chronic liver diseases. J Hepatol 47: 598-607, 2007.

19. Smith TA, Kirkpatrick DR, Smith S, Smith TK, Pearson T, Kailasam A, Herrmann KZ, Schubert J and Agrawal DK: Radioprotective agents to prevent cellular damage due to ionizing radiation. J Transl Med 15: 232, 2017.
20. Tan DX, Reiter RJ, Manchester LC, Yan MT, El-Sawi M, Sainz RM, Mayo JC, Kohen R, Allegra M and Hardeland R: Chemical and physical properties and potential mechanisms: Melatonin as a broad spectrum antioxidant and free radical scavenger. Curr Top Med Chem 2: 181-197, 2002.

21. Koc M, Taysi S, Buyukokuroglu ME and Bakan N: Melatonin protects rat liver against irradiation-induced oxidative injury. J Radiat Res 44: 211-215, 2003.

22. Taysi S, Koc M, Büyükokuroğlu ME, Altınkaynak K and Sahin YN: Melatonin reduces lipid peroxidation and nitric oxide during irradiation-induced oxidative injury in the rat liver. J Pineal Res 34: 173-177, 2003.

23. Reiter RJ, Tan DX, Osuna C and Gitto E: Actions of melatonin in the reduction of oxidative stress. A review. J Biomed Sci 7: 444-458, 2000.

24. Vijayalaxmi, Reiter RJ and Meltz ML: Melatonin protects human blood lymphocytes from radiation-induced chromosome damage. Mutat Res 346: 23-31, 1995.

25. Sharma S, Haldar C, Chaube SK, Laxmi T and Singh SS: Long-term melatonin administration attenuates low-LET gamma-radiation-induced lymphatic tissue injury during the reproductively active and inactive phases of Indian palm squirrels (Funambulus pennanti). Br J Radiol 83: 137-151, 2010.

26. Sharma S, Haldar C and Chaube SK: Effect of exogenous melatonin on X-ray induced cellular toxicity in lymphatic tissue of Indian tropical male squirrel, Funambulus pennanti. Int J Radiat Biol 84: 363-374, 2008.

27. Sener G, Jahovic N, Tosun O, Atasoy BM and Yeğen BÇ: Melatonin ameliorates ionizing radiation-induced oxidative organ damage in rats. Life Sci 74: 563-572, 2003.

28. Kucuktulu E: Protective effect of melatonin against radiation induced nephrotoxicity in rats. Asian Pac J Cancer Prev 13: 4101-4105, 2012.

29. Karslioglu I, Ertekin MV, Taysi S, Koçer I, Sezen O, Gepdiremen A, Koç M and Bakan N: Radioprotective effects of melatonin on radiation-induced cataract. J Radiat Res 46: 277-282, 2005.

30. Haddadi G, Shirazi A, Sepehrizadeh Z, Mahdavi SR and Haddadi M: Radioprotective effect of melatonin on the cervical spinal cord in irradiated rats. Cell J 14: 246-253, 2013.

31. Erol FS, Topsakal C, Ozveren MF, Kaplan M, Ilhan N, Ozercan IH and Yildiz OG: Protective effects of melatonin and vitamin E in brain damage due to gamma radiation: An experimental study. Neurosurg Rev 27: 65-69, 2004.

32. Vijayalaxmi, Meltz ML, Reiter RJ, Herman TS and Kumar S: Melatonin and protection from whole-body irradiation: Survival studies in mice. Mutat Res 425: 21-27, 1999.

33. Davis TA, Clarke TK, Mog SR and Landauer MR: Subcutaneous administration of genistein prior to lethal irradiation supports multilineage, hematopoietic progenitor cell recovery and survival. Int J Radiat Biol 83: 141-151, 2007. 